\title{
Research Status of Cloud Manufacturing Resources
}

\author{
Wang Zhanli \\ Changchun University of Technology \\ Changchun, China \\ Hu Yanjuan* \\ Changchun University of Technology \\ Changchun, China \\ E-mail:yanjuan_hu@126.com \\ *Corresponding Author
}

\begin{abstract}
In order to analyze the current situation of CMR (cloud manufacturing resources) after the human entering to net work age and cloud manufacturing formally producing, then summarizing and analyzing the related work on cloud manufacturing and CMR, three aspects of CMR are analyzed from the classification, modeling and virtualization. It shows that the study of CMR has made many research results that make a contribution to cloud manufacturing in such a short period of time, but these researches are not only in the primary stage, but they are also not concentrated or uniform. Therefore, after reading related research about the existing CMR, some key problems in the research based on CMR are put forward in these aspects, in the future, that solving key issues will provide the necessary support for the development of cloud manufacturing.
\end{abstract}

Keywords-CMR; classification; modeling; virtualization; key problems

\section{INTRODUCTION}

The manufacturing industry is an important pillar of the development of human society, its development reflects a country or region's economy and the level of comprehensive strength. Since the mankind entered the information age, the manufacturing industry has undergone enormous changes because of the influence of knowledge economy, globalization thinking, network economy and technological innovation. Cloud manufacturing is born at this time, along with the development of manufacturing industry, the definition of manufacturing resources also gives the time significance. Generalized manufacturing resources is the product of the entire life cycle of all production activities of the software and hardware elements, including the design, manufacturing, maintenance and other related activities involved in the process of all the elements. Manufacturing resources in narrow sense mainly refers to the material elements needed for processing a part, it is a manufacturing resource for the underlying manufacturing system, it mainly includes machine tools, cutting tools, fixtures, measuring tools and materials, etc. Therefore, the research of CMR plays an important role in the development of cloud manufacturing.

\section{CMR}

B.H.Li [1], academician, first formally defined cloud manufacturing--create a new model of Chinese.

\author{
Chang Xingfu \\ Changchun University of Technology \\ Changchun, China
}

Cloud manufacturing integrate cloud computing, networking, information technology and high performance computing technology, virtualization, service and other resources, to provide users with high quality, low cost and security service of network manufacturing new models in product life cycle. CMR are collecting all kinds of physical factors in the product life cycle, it refers to everything that can play a role in the entire product life cycle, the completion of all production and manufacturing activities involved in the software and hardware resources (including equipment, materials, etc.), human (technical experts, the general operation of workers, etc.), knowledge (technical information, logistics information and related data, etc.), and so on.

The characteristics of CMR are different from the conventional manufacturing resources.

\section{A. Distribution and heterogeneity}

CMR is not centralized but distributed in different geographical locations and organizations, the variety of CMR is varied in cloud manufacturing environment. All these manufacturing resources management, organization, sharing and specifications in enterprises and factories are not the same, however, integrated in their respective management system. Therefore, it has the characteristics of distribution and heterogeneity.

\section{B. Diversity and dynamic}

CMR is not only a wide variety, but also belong to different companies and organizations. With the changes and development of manufacturing activities, environment, technology, and so on, CMR can provide the service and the ability of the users can be changed dynamically. The original manufacture resources available over time may become unusable, making new resources will gradually join in, which reflects the dynamic nature of manufacturing resources.

\section{Abstraction and consistency}

CMR makes all the resources highly abstract for the user visible "power supply wiring board", other things are transparent to the user. CMR is unified and coordinated by the platform of cloud manufacturing. CMR in different regions and different environments can be consistent through the reasonable definition of resources. 


\section{STATUS AND EXISTING PROBLEMS OF CMR}

Since the concept of cloud manufacturing was put forward, many scientific research workers have carried out many researches and studies on CMR, which mainly focus on the classification, modeling and virtualization.

\section{A. Related work about classification based on CMR}

With the increasing number of CMR and basic position of manufacturing resources in cloud manufacturing system, so it is very important to classify it scientifically and reasonably. Different kinds of manufacturing resources can be obtained by different perspectives and classification methods. According to different research directions, many scholars have made a detailed analysis of the types of manufacturing resources, as shown in Table 1.

TABLE 1 CLASSIFICATION BASED ON CMR

\begin{tabular}{|l|l|}
\hline Author & Classification based on CMR \\
\hline Q.N.Yuan [2] & Classify into equipment, enterprise resources, software resources, \\
human resources, standard resources, product resources, service \\
resources \\
Classify resources into the equipment, material, human, knowledge, \\
Z.C.Wang [3] \\
service, software, information \\
Equipment, information resources, software resources, precision \\
instruments, high performance computer resources, technology \\
resources, information resources, user resources, \\
Human resources, equipment resources, technical resources, material \\
B.Y.Sheng [5] \\
resources, application system resources, user information resources, \\
service resources, computing resources
\end{tabular}

\section{B. Research status of modeling on CMR}

Modeling on CMR is based on the characteristics and classification. At present, the main methods of modeling are object-oriented method and modeling method based on ontology.

After consulting existing status about modeling based on CMR, the current situation of manufacturing resource modeling was summarized. Z.C.Wang [6] proposed a process model of cloud manufacturing service driven by temporal constraint. S.Zhang [7] proposed the resource selection based on workflow. A semantic modeling of manufacturing resources and manufacturing capabilities to form a cloud service, and build a cloud manufacturing platform based on Ontology by Z.N.Zhang [8]. C.Yin [9] built up the service capability model, the service demand model and the supply and demand model of the enterprise for the medium and small-sized enterprises. Resource allocation model based on cost and time minimization and quality optimization is constructed by S.L.Wang [10], solving the model with maximum succession law.

\section{Research status of virtualization on CMR}

Virtualization based on CMR is the integration and sharing CMR, the perception and feedback control through the use of Internet technology, cloud computing virtualization and other technologies, transforming manufacturing resources into virtual manufacturing services. Through the reasonable scheduling of resources, in manufacturing service environment.

The framework of virtualization on CMR is divided into five levels by L.Ren [11]: manufacturing resources, the Internet of things /CPS infrastructure, physical resources, the pool of virtual resource and virtual resource management, and it also puts forward the key technology of cloud manufacturing based on virtual support. L.Wu [12] proposed a model about resource virtualization in cloud manufacturing environment, including the manufacturing resource layer, Web service layer, logical service layer and application layer. X.F.Yao [13] discussed the principle of cloud manufacturing resource virtualization--system, pertinence, adaptability, scalability, interface and dynamic, taking manufacturing equipment resource as an example, analyzing the virtual description model and giving the virtual template of manufacturing equipment resource.

\section{Existing problems}

So far, the research on CMR is at the beginning stage. Many researchers have carried out the research on the classification, modeling and virtualization of CMR, and have made some contributions to the development of CMR. But in the summary of the results of their research, there are still many problems that need to be studied.

\section{1) Classification on CMR}

There is no uniform rule or standard to define resource classification, it leads that classification is not in the normal way in the bottom of the cloud manufacturing. This will have a great impact on the future of research about classification, modeling, virtualization based on CMR. Therefore, in order to achieve the unification and standardization of classification, the key is building a reasonable classification standard.

\section{2) Modeling on $C M R$}

Many researchers have done a lot of research on modeling, but it's only at the theoretical level, and it haven't realize how to apply the model to the actual operation of the enterprise. So the key of modeling is to connect the model to the physical resources in a right and reasonable way, and to develop a more reasonable modeling method.

\section{3) Virtualization on CMR}

The study of virtualization is, only at the surface and the theoretical level, studying that the concept, framework and model of resource virtualization, for example, how to realize the integration of the virtual resources and the bottom equipment, material resources and enterprise resources, how to realize the connection between the 
virtual modeling of manufacturing resources and the cloud virtualization resources model.

\section{CONCLUSION AND FUTURE WORK}

In conclusion, the research on CMR is still in its early stages. With the government's high degree of attention and strong support, as well as the relevant scholars to study hard, the research about CMR will gradually mature. Its maturity will provide the necessary support for the development of cloud manufacturing, Eventually, it will promote the intelligent development of cloud manufacturing industry.

\section{ACKNOWLEDGMENT}

This research work was supported by the Nature Science Foundation of China, and the project name is "Research on theory and method of manufacturability evaluation in cloud manufacturing environment", no. 51405030.

\section{REFERENCE}

[1] [1] B.H.Li, L.Zhang, S.L.Wang, "Cloud Manufacturing: A New Service-Oriented Manufacturing Model," Computer Integrated Manufacturing Systems, vol.16, pp.1-7, 2010.

[2] [2] Q.N.Yuan, "Research on manufacturing resources ontology model based on semantic," Journal of Wuhan university of technology, vol.31, pp.121-125, May.2009.

[3] [3] Z.C.Wang, "Formalized Model on Networked Manufacturing Resource and Realization," Journal of Zhejiang Sci-Tech University, vol.26, pp.705-710, 2009.
[4] [4] F.Liang, "Model building approach of manufacturing resource based on meta resource" Computer Integrated Manufacturing Systems, vol.14, pp2306-2311, 2008.

[5] [5] B.Y.Sheng, "Modeling and Management of Manufacturing Resource Information in Manufacturing Grid,” China Mechanical Engineering, vol.17, pp.1375-1380, 2006.

[6] [6] Z.C.Wang, Y.Huang, "Research on Integrated Sharing Technology of Cloud Manufacturing Resource Oriented to Service Chain Construction," China Mechanical Engineering, vol23, pp.1324-1331, 2012.

[7] [7] S.Zhang, "Resource Selection Method Based on Workflow in Cloud Manufacturing," Computer Integrated Manufacturing Systems, vol.21, pp.831-839, 2015.

[8] [8] Z.N.Zhang, "Key Issues for Cloud Manufacturing Platform," Advance Materials Research, vol.472, pp.2621-2625, 2012.

[9] [9] C.Yin, B.Q.Huang, F.Liu, “Common Key Technology System of Cloud Manufacturing Service Platform for Small and Medium Enterprises," Computer Integrated Manufacturing Systems,vol.17,pp.495-503,2011.

[10] [10] S.L.Wang, W.Y.Song, L.Kang, "Manufacturing Resource Allocation Based on Cloud Manufacturing," Computer Integrated Manufacturing Systems, vol.18, pp.1396-1405, 2012.

[11] [11] L.Ren, L.Zhang, "Resource Virtualization in Cloud Manufacturing," Computer Integrated Manufacturing Systems, vol.17, pp.511-518, 2011.

[12] [12] L.Wu, "Resource Virtualization Model in Cloud Manufacturing," Advance Materials Research, vol.(143-144), pp.1250-1253, 2011.

[13] [13] X.F.Yao, H.Jin, C.Xu, "Virtualization and Servitization of Cloud Manufacturing Resources," Journal of South China University of Technology(Natural Science Edition), vol.41, pp.1-7, 2013 